\title{
Diagnóstico e tratamento do lenticone anterior
}

\section{Diagnosis and therapy of anterior lenticonus}

\author{
Ana Luiza Biancardi', Renato Ambrosio Júnior ${ }^{2}$, Aileen Walsh ${ }^{3}$, Armando Stefano Crema ${ }^{4}$
}

\section{Resumo}

Relato de caso de um paciente de 18 anos com sindrome de Alport apresentando perda visual progressiva. A biomicroscopia revelou lenticone anterior bilateral. O paciente realizou tomografia de córnea e segmento anterior com o sistema Scheimpflug (Pentacam) e aberrometria e topografia corneana (i-Trace). O paciente foi submetido à facoemulsificação com implante de lente intraocular peça única hidrofóbica (Acrysof ${ }^{\circledR}$ SN60AT). As imagens de Scheimpflug documentaram o lenticone anterior. A aberrometria total mostrou acentuado astigmatismo miópico com acentuada aberração esférica negativa, havendo grande impacto das aberrações de alta ordem (HOA) na conversão da letra E de Snellen. O mapa de integração da aberrometria do olho todo com a topografia corneana mostrou maior semelhança das aberrrações totais com as aberrações intraoculares do que com as aberrações da superfície anterior da córnea. Após a cirurgia, o paciente apresentou acuidade visual corrigida igual a 20/20 em ambos os olhos, as imagens de Scheimpflug revelaram lentes intraoculares tópicas e os mapas diferenciais revelaram resolução da miopia e redução das aberrações da alta ordem (total e interna). Os exames de imagem foram úteis para demonstrar o impacto do lenticone anterior na qualidade visual e a resolução das aberrações ópticas após a cirurgia.

Descritores: Nefrite hereditária/complicações; Manifestações oculares/terapia; Facoemulsificação; Implante de lente intraocular; Relatos de casos

\section{Abstract}

Case report of a 18-year-old male patient with Alport's syndrome, who presented with renal failure and hearing loss reporting a progressive loss of vision acuity. Slitlamp examination revealed bilateral anterior lenticonus. The patient underwent pre and post-operative corneal and anterior segment tomography with a rotating Scheimpflug system (Pentacam), integrated whole eye (individual laser beam ray tracing) and corneal surface (Placido) wavefront aberrometry (i-Trace). Phacoemulsification and single piece hydrophobic IOL implantation (Acrysof® SN60AT) was performed uneventfully in both eyes. Pre op Scheimpflug well decomented the anterior lenticonus. Total aberrometry showed myopic astigmatisms with high negative spherical aberrations $O U$; higher order aberrations (HOAs) greatly influenced the conversion of Snellen E letters. Total aberrations and corneal topographies integration maps OU demonstrated high levels of intraocular aberrations which had more similarity with whole eye aberrations than anterior corneal aberrations. After surgery, BCVA was 20/20 in both eyes. Scheimpflug images revealed topic intraocular lens and the differential maps showed resolution of myopia and decrease of the total ocular HOAs as well as from the internal optics. Adjunctive advanced diagnostic tools were useful to demonstrate the anterior lenticonus and its optical impact along with the resolution of the aberrations post operatively. Case reports

Keywords: Nephritis, hereditary/complications; Eye manifestations; Phacoemulsification; Lens implantation, intraocular;

\footnotetext{
${ }^{1}$ Mestre em Oftalmologia pela Universidade Federal do Rio de Janeiro - UFRJ -- Rio de Janeiro (RJ), Brasil;

${ }^{2}$ Doutor em Oftalmologia pela Universidade de São Paulo - USP - São Paulo (SP), Brasil;

${ }^{3}$ Mestre em Oftalmologia pela Universidade Federal do Rio de Janeiro - UFRJ - Rio de Janeiro (RJ), Brasil;

${ }^{4}$ Professor Assistente de Oftalmologia da Universidade Gama Filho - UGF - Rio de Janeiro (RJ), Brasil.
}

Instituições:

Walsh \& Crema Clínica e Microcirurgia Ocular / Visare Personal Laser - Rio de Janeiro (RJ), Brasil

The autors received no public or private financial support

Recebido para publicação em: 24/8/2010 - Aceito para publicação em 8/2/2011 


\section{INTRODUÇÃO}

A síndrome de Alport é uma doença progressiva hereditária com comprometimento renal, auditivo e oftalmológico. Mutações envolvendo a cadeia alfa-5 do colágeno tipo IV, encontrado nas membranas basais do glomérulo, cóclea, saco capsular e retina, estão associadas a esta síndrome ${ }^{(1)}$.

As alterações oculares geralmente acometem o cristalino e se manifestam por lenticone anterior, catarata subcapsular posterior e esferofacia ${ }^{(1)}$.

O lenticone anterior é uma rara anormalidade do cristalino que resulta em visão subnormal progressiva e incapacitante, cujo tratamento cirúrgico requer especial atenção devido à fragilidade do saco capsular ${ }^{(2,3)}$.

Anormalidades do cristalino estão relacionadas com aberrações ópticas internas e provocam redução da qualidade visual. A comparação entre a aberração ocular total medida por um aberrômetro e as aberrações corneanas anteriores medidas pela topografia corneana permitem a separação das aberrações corneanas daquelas relacionadas ao cristalino(4).

O objetivo do presente relato é descrever as aberrações ópticas relacionadas ao lenticone anterior bilateral em um paciente com síndrome de Alport e a resolução das aberrações após a facoemulsificação com implante de lente intraocular.

\section{Relato de caso}

Paciente do sexo masculino, 18 anos, portador de síndrome de Alport com hipoacusia e insuficiência renal, procurou atendimento com queixa de perda progressiva da acuidade visual. Apresentou ao exame oftalmológico acuidade visual corrigida igual a 20/200

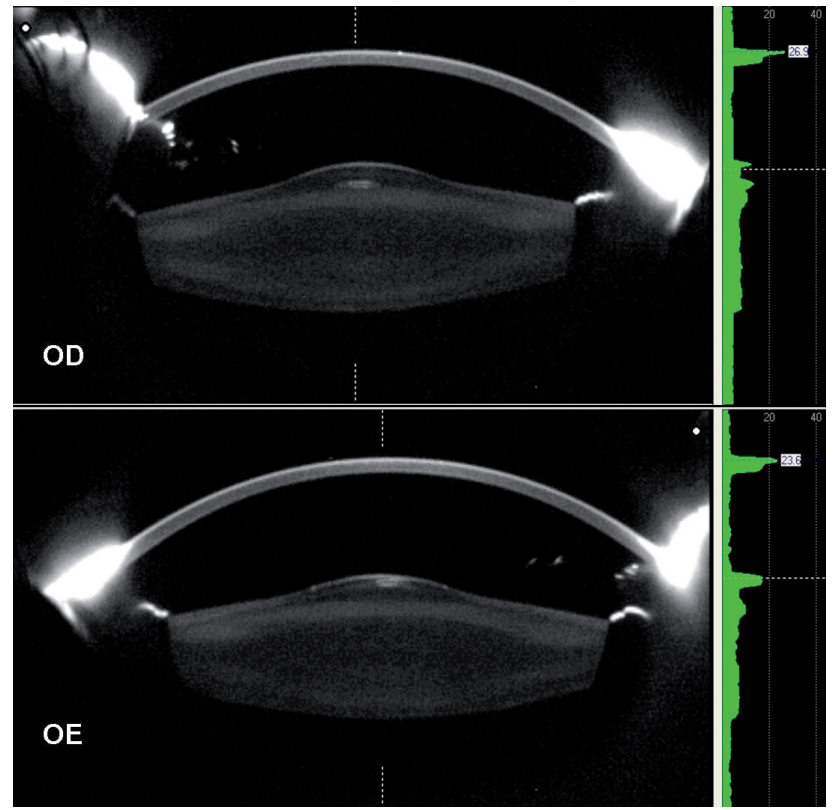

Figura 1: (pré-operatório) Tomografia de segmento anterior com imagens de Scheimpflug (Pentacam) do OD e OE, demonstrando o lenticone anterior

no olho direito (OD) e 20/100 no olho esquerdo (OE). A biomicroscopia revelou lenticone anterior bilateral. A pressão intraocular e o fundo de olho não apresentaram alterações em ambos os olhos (AO).

Foram realizadas tomografia da córnea e segmento anterior por fotografias Scheimpflug rotacionais (Pentacam) e aberrometria (i-Trace).

As imagens de Scheimpflug documentaram o lenticone anterior (figura 1) e mostraram área de scattering maior que $21 \% \mathrm{AO}$, com média de scattering igual a $9,8 \%$ no OD e $7,1 \%$ no OE.

A aberrometria total mostrou astigmatismo miópico com aberrações esféricas negativas de alta ordem em AO.
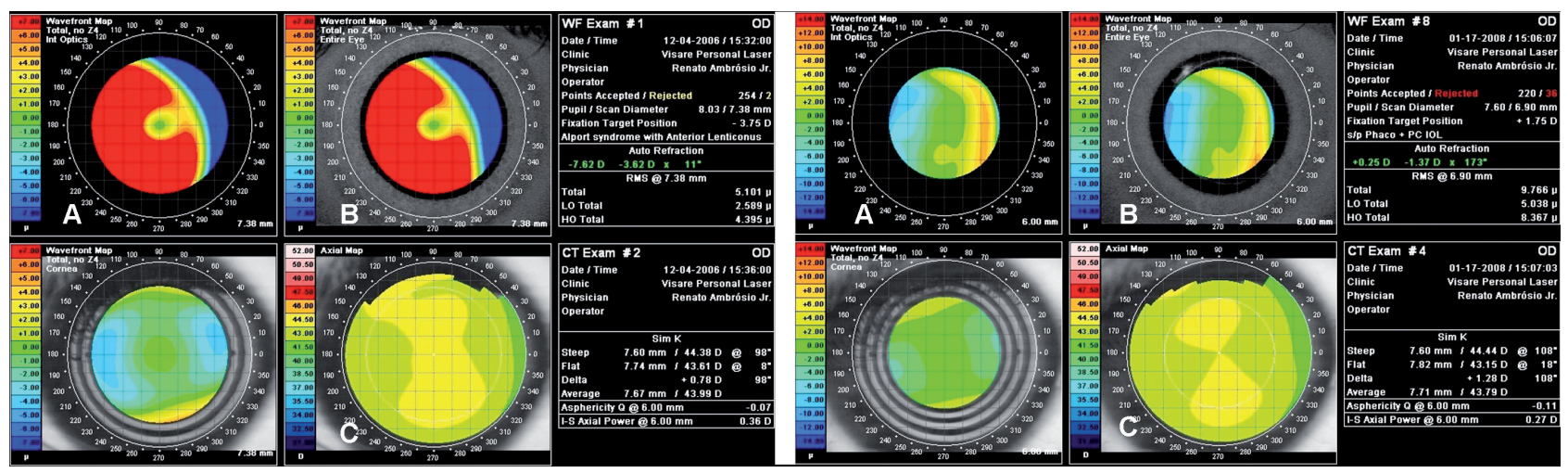

Figura 2: Aberrometria (i-Trace) do OD; (esquerda): pré-operatório; (direita) pós-operatório; A) Aberrometria total; B) Aberrações intraoculares; C) Aberrações corneanas anteriores; Os mapas diferenciais revelaram resolução da miopia e redução das aberrações de alta ordem (total e interna) 
A aberrometria demonstrou que as aberrações totais se assemelhavam mais às aberrações intraoculares do que às aberrações anteriores. (Figuras 2 e 3 )

Foi realizada facoemulsificação com implante de lente intraocular hidrofóbica de peça única (Acrysof ${ }^{\circledR}$ SN60AT) sem intercorrências em AO.

Após a cirurgia, o exame oftalmológico revelou acuidade visual igual a 20/20 em AO e foi repetida a análise do segmento anterior. As imagens de Scheimpflug revelaram lentes intraoculares tópicas (figura 4) e os mapas diferenciais revelaram resolução da miopia e redução das aberrações de alta ordem (total e interna). As aberrações corneanas permaneceram estáveis (figuras 2 e 3 ).

\section{Comentários}

O diagnóstico da síndrome de Alport está relacionado à história familiar positiva de hematúria com ou sem insuficiência renal, biópsia da membrana basal glomerular com alterações características à microscopia eletrônica, comprometimento oftalmológico e surdez neurossensorial ${ }^{(1)}$

As manifestações oftalmológicas na síndrome de Alport podem envolver a córnea, a retina ou o cristalino. $\mathrm{Na}$ córnea, dois achados frequentes são a distrofia polimorfa posterior e o arco senil. Na retina o achado mais comum são flecks na mácula ou meia periferia, sem impacto na acuidade visual. No cristalino foram descritos esferofacia, catarata e coloboma, no entanto o lenticone anterior é a alteração mais comum, e acomete principalmente homens durante a segunda e terceira décadas de vida ${ }^{(1)}$.

Apesar de o lenticone ser facilmente diagnosticado ao exame biomicroscópico, este não permite uma análise quantitativa do segmento anterior, que pode ser

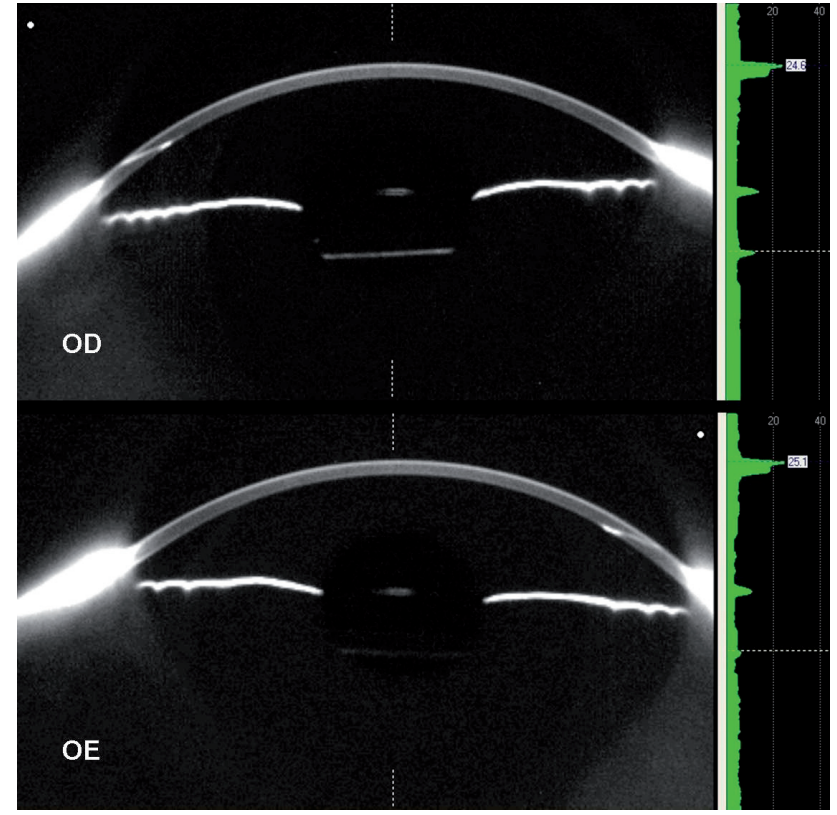

Figura 3: Aberrometria (i-Trace) do OE; (esquerda): pré-operatório; (direita) pós-operatório;A) Aberrometria total;B) Aberrações intraoculares; C) Aberrações corneanas anteriores. Os mapas diferenciais revelaram os mesmos dados do OD

realizada através das imagens de Scheimpflug ${ }^{(5)}$. No presente relato, os exames complementares de imagem foram úteis para documentar o lenticone anterior, o impacto na qualidade visual relacionado a esta anormalidade do cristalino e a resolução das aberrações ópticas após a cirurgia de facoemulsificação.

Relatos prévios ${ }^{(2,3,6)}$ avaliaram a cápsula anterior do lenticone através da microscopia eletrônica e demonstraram afinamento e deiscência contendo vacúolos e material fibrilar, características responsáveis pela fragilidade desta estrutura (o que dificulta a capsulorrexe) e relatos de ruptura espontânea da cápsula anterior na evolução do lenticone ${ }^{(7,8)}$. No nosso caso, não tivemos
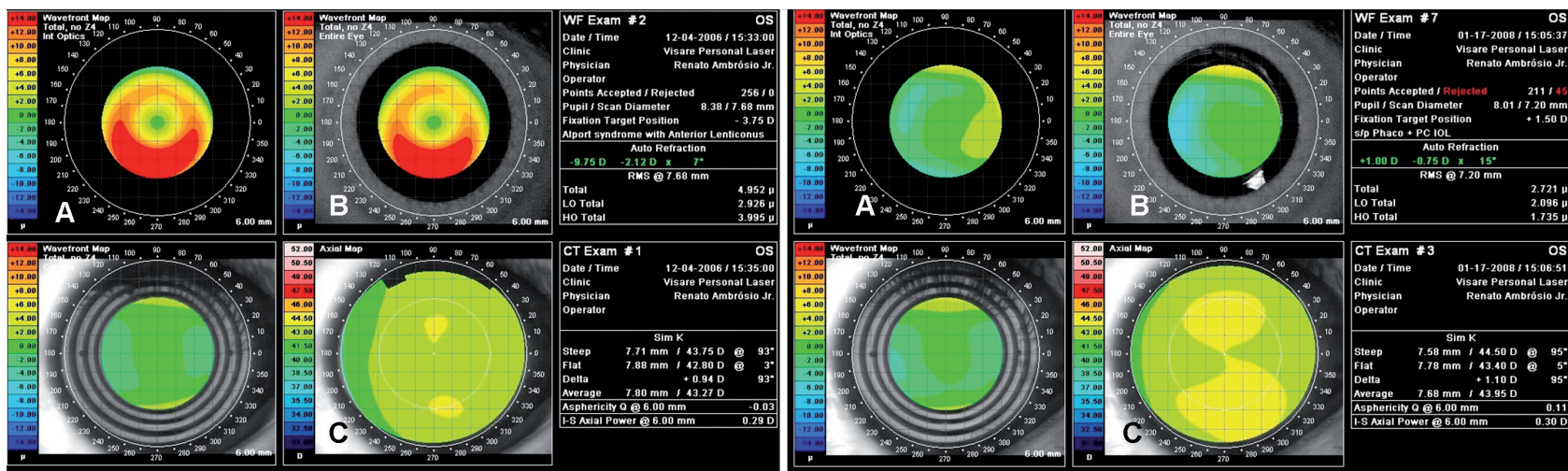

Figura 4: (pós-operatório) Tomografia de segmento anterior com imagens de Scheimpflug (Pentacam) do OD e OE, demonstrando lentes intraoculares tópicas 
intercorrências durante a cirurgia apesar da dificuldade na realização da capsulorrexe.

Embora o lenticone esteja relacionado à fragilidade da cápsula anterior, nosso relato sugere que a facoemulsificaçao é um procedimento seguro e eficaz para a recuperação da acuidade e qualidade visual em pacientes portadores de síndrome de Alport e lenticone anterior.

\section{REFERÊNCIAS}

1. McCarthy PA, Maino DM. Alport syndrome: a review. Clin Eye Vis Care. 2000;12(3-4):139-50.

2. Junk AK, Stefani FH, Ludwig K. Bilateral anterior lenticonus: Scheimpflug imaging system documentation and ultrastructural confirmation of Alport syndrome in the lens capsule. Arch Ophthalmol. 2000;118(7):895-7.

3. van Setten G. Anterior lenticonus: histological evaluation and approach for cataract surgery. J Cataract Refract Surg. 2001;27(7):1071-5.

4. Kruger RR, Applegate RA, Macrae SM. Wavefront customized visual correction: the quest for Super Vision II. New Jersey: Slack; 2004.
5. Zhou W, Hirsch M, Junk AK, Casper DS, Braunstein R, David J, Worgul BV. Evaluation of lenticonus in Alport's syndrome: quantitative Scheimpflug analysis. Ophthalmologica. 2003;217(3):189-93

6. Oliveira A, Rezende Filho F, Rezende F. Faco-tópica na Síndrome de Alport com lentecone anterior: avaliação pré, per e pós-operatória. Rev Bras Oftalmol. 2000;59(2):91-6.

7. Sathish KR, Chandrashekar N, Pai V, Bhatia P, Kakkar G. Spontaneous capsular ruptures in Alport syndrome. Ann Ophthalmol. 2001;33(2):131-5.

8. Cresta FB, Silva ALB, Carvalho CA. Ruptura espontânea da cápsula anterior do cristalino em paciente com síndrome de Alport. Arq Bras Oftalmol. 1998;61(6):717-9.

Endereço para Correspondência:

Ana Luiza Biancardi

Rua Vilhena de Moraes, $n^{\circ}$ 100, apto. 903, bloco 02 -

Barra da Tijuca

Rio de Janeiro - (RJ), Brasil

Fax: (21) 2431-1355

albiancardi@ig.com.br 